Rev. Int. Contam. Ambie. 34 (1) 35-43, 2018

DOI: $10.20937 /$ RICA.2018.34.01.03

\title{
COMPORTAMIENTO DE EVASIÓN Y REPRODUCCIÓN DE LA LOMBRIZ Eisenia foetida EN SUELOS AGRÍCOLAS IMPACTADOS POR ACTIVIDADES MINERAS
}

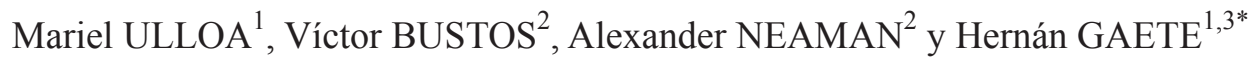

${ }^{1}$ Escuela de Ingeniería en Medioambiente, Facultad de Ingeniería, Universidad de Valparaíso, General Cruz
222 , Valparaíso, Chile
${ }^{2}$ Escuela de Agronomía, Pontificia Universidad Católica de Valparaíso, La Palma s/n, Quillota, Valparaíso,
Chile
${ }^{3}$ Centro de Investigación y Gestión de Recursos Naturales (CIGREN), Av. Gran Bretaña 1111, Playa Ancha,
Valparaíso, Chile
*Autor de correspondencia; hernan.gaete@uv.cl

(Recibido septiembre 2016; aceptado mayo 2017)

Palabras clave: toxicidad, cobre, metales

\section{RESUMEN}

El objetivo de este estudio fue determinar la toxicidad de suelos agrícolas cercanos a zonas con actividad minera sobre la lombriz Eisenia foetida. Con este fin se determinaron en los suelos colectados las concentraciones totales de los metales $\mathrm{As}, \mathrm{Cu}, \mathrm{Zn}$, $\mathrm{Pb}$, y solubles de $\mathrm{As} \mathrm{y} \mathrm{Cu}, \mathrm{pCu}^{+2}$, granulometría, materia orgánica, conductividad y $\mathrm{pH}$. La toxicidad se determinó a través de bioensayos agudos de conducta de evasión y crónicos de reproducción. Los resultados muestran que las concentraciones totales de estos metales en los suelos tienen el siguiente orden decreciente: $\mathrm{Cu}>\mathrm{Zn}>\mathrm{Pb}>$ As. De los 14 suelos estudiados, los dos que presentaron las mayores concentraciones de cobre total afectaron el comportamiento de evasión de E. foetida. Por otra parte, los suelos con mayor porcentaje de materia orgánica y arcilla presentaron los menores porcentajes de evasión. La producción de capullos fue similar en la mayoría de los suelos, a excepción de tres, en los que fue significativamente menor $(\mathrm{p}<0.05)$, en tanto que la producción de juveniles - con excepción de un suelo - no exhibió variación significativa $[p>0.05])$. La producción de capullos y juveniles se relacionó inversamente con las concentraciones de cobre total y soluble. En conclusión, la mayoría de los suelos no contiene agentes químicos biodisponibles que provoquen toxicidad o afecten la capacidad de selección del hábitat ni la reproducción en $E$. foetida. Las variables de comportamiento de evasión y reproducción se relacionaron inversamente. Se sugiere el uso alternativo de bioensayos de comportamiento de evasión, como herramienta de alerta temprana en el monitoreo de suelos que puedan contener agentes tóxicos potenciales.

Key words: toxicity, copper, metals

\begin{abstract}
The aim of this study was to determine the toxicity of agricultural soils in areas with mining activities on worm Eisenia foetida. Total concentrations of $\mathrm{As}, \mathrm{Cu}, \mathrm{Zn}, \mathrm{Pb}$, and soluble $\mathrm{As}$ and $\mathrm{Cu}, \mathrm{pCu}^{+2}$, granulometry, organic matter, $\mathrm{pH}$ and conductivity in the
\end{abstract}


collected soil were determined. Toxicity was determined using bioassays of avoidance behavior and reproduction. The results show that the total concentrations of metals in soil have the following decreasing order: $\mathrm{Cu}>\mathrm{Zn}>\mathrm{Pb}>\mathrm{AS}$. Of the 14 soils studied, the two with highest concentrations of total copper had effect on the avoidance behavior of $E$. foetida. Soils with the highest percentage of organic matter and clay caused the lowest percentages of avoidance behavior. The production of cocoons was similar in most soils, except three in which it was significantly lower $(\mathrm{p}<0.05)$; regarding juvenile production no significant variation $(\mathrm{p}>0.05)$ was observed, except for one soil. These variables were inversely correlated to the concentration of total and soluble copper. In conclusion, most soils do not contain bioavailable chemical agents that provoke toxicity or affect the capacity of $E$. foetida both for habitat selection or reproduction. Avoidance behavior and reproduction were inversely related. Reproduction decreased in soils where evasion was higher. So the alternative use of bioassays of avoidance behavior, as an early warning tool, is suggested for monitoring soils that may contain potential toxic agents.

\section{INTRODUCCIÓN}

La contaminación de suelos agrícolas con metales provenientes de las actividades antrópicas como la minería ha aumentado en los últimos años (Hormazábal et al. 2013). Si bien algunos metales como el cobre son nutrientes esenciales, forman parte de la estructura de proteínas y participan en diversas reacciones metabólicas (Navarro-Aviñó et al. 2007, Ferreira-Cravo et al. 2009), en altas concentraciones pueden ser tóxicos para los animales y vegetales que habitan el suelo (Barros-Amorim y Scott-Fordsmand 2012, Rodríguez et al. 2015). Entre estos se encuentran las lombrices de tierra, organismos que cumplen un rol fundamental en la descomposición de la materia orgánica, el ciclo de nutrientes y la aireación del suelo.

En la evaluación del riesgo ambiental de contaminantes ambientales se deben establecer tanto la exposición como los efectos sobre los organismos. La exposición tradicionalmente se determina mediante la estimación de concentraciones de los agentes químicos en el ambiente; sin embargo, ésta no es suficiente para establecer su biodisponibilidad y por lo tanto los potenciales efectos tóxicos. Por lo anterior, la determinación del efecto de estos agentes químicos sobre los organismos del suelo se lleva a cabo por medio de bioensayos de toxicidad agudos y crónicos de mortalidad, reproducción y crecimiento de la lombriz de tierra Eisenia foetida (Ávila et al. 2007), entre otras especies. Estos bioensayos de toxicidad crónicos requieren mucho tiempo (56 días) y son costosos (Udovic y Lestan 2010). Como alternativa, se ha propuesto el bioensayo de conducta de evasión con la lombriz E. foetida, (ISO 2007, Žižek y Zidar 2013). Esta prueba se usa como herramienta de monitoreo de alerta temprana de toxicidad de suelos, ya que permite evaluar en corto tiempo (48 h) el efecto de éstos sobre la capacidad de las lombrices para seleccionar un hábitat para sus procesos biológicos y ecológico. La variable respuesta de evasión se basa en la presencia de quimiorreceptores en el prostomio y en el segmento anterior, así como la distribución de tubérculos a lo largo del cuerpo, los cuales hacen a las lombrices muy sensibles a los agentes químicos en el ambiente y les permiten evitar condiciones ambientales desfavorables gracias a su capacidad locomotriz (Curry y Schmidt 2007, Udovic y Lestan 2010).

La cuenca del río Aconcagua en la región de Valparaíso (Chile central), se caracteriza por tener importantes asentamientos urbanos, además de ser una zona de gran relevancia agrícola y minera (MOP 2004). En algunas áreas de la cuenca, los suelos de uso agrícola presentan elevados niveles de metales como cobre y plomo, los cuales estarían asociados a las emisiones proveniente de fundiciones y termoeléctricas (Aguilar et al. 2011, Ginocchio et al. 2013, González et al. 2014). Los estudios de toxicidad en esta zona se han realizado mediante bioensayos de toxicidad de reproducción, crecimiento y biomarcadores moleculares. Entre ellos destacan los trabajos realizados por Ávila et al. $(2007,2009)$ y Cataldo et al. (2011). Sin embargo, se desconoce si estos suelos contienen agentes químicos que pudieran afectar la capacidad de selección del hábitat de las lombrices de tierra, lo que a su vez comprometería su reproducción. Por lo anterior, el objetivo de esta investigación fue determinar la toxicidad de suelos agrícolas en la cuenca del Río Aconcagua a través de bioensayos de toxicidad aguda de comportamiento de evasión y crónico de reproducción de lombriz 
E. foetida, y relacionarlos con las características físicas y químicas de los suelos estudiados.

\section{MATERIALES Y MÉTODOS}

\section{Área de estudio}

En este estudio se analizaron suelos superficiales de áreas agrícolas históricamente contaminadas por las actividades mineras en Chile. Los puntos de muestreo fueron seleccionados con base en el conocimiento previo sobre la distribución espacial del $\mathrm{Cu}$ en los suelos agrícolas de la cuenca del río Aconcagua (Aguilar et al. 2011) y el Valle de Puchuncaví (González et al. 2014). Los sitios y puntos de muestreo se eligieron con el objetivo de obtener un gradiente de concentraciones totales de metales (Fig. 1, Cuadro I). En cada punto de muestreo se tomaron muestras de suelo en una superficie de $0.5 \mathrm{~m}^{2}$ hasta una profundidad de $20 \mathrm{~cm}$. Las muestras de suelo se transportaron en bolsas plásticas al laboratorio para su caracterización física y química, y la realización de bioensayos de toxicidad (Cuadro I). Las muestras se tamizaron a través de una malla de $2 \mathrm{~mm}$ y luego se homogenizaron en un mezclador de cemento con cubierta de plástico. Posteriormente, se secaron en horno a una temperatura interior de $40{ }^{\circ} \mathrm{C}$ durante $48 \mathrm{~h}$. Con el fin de verificar la homogeneidad de los suelos mezclados, se obtuvieron y se analizaron cuatro submuestras de cada muestra.

\section{Caracterización física y química de los suelos}

La conductividad eléctrica se midió en solución 1:5 de suelo:agua. Los valores de $\mathrm{pH}$ y $\mathrm{pCu}^{+2}$ se midieron en solución de $0.1 \mathrm{M} \mathrm{KNO}_{3}(1: 2.5$ suelo:solución) de acuerdo con Sadzawka et al. (2006). La determinación de $\mathrm{pCu}^{2+}\left(\mathrm{pCu}^{2+}=-\log \right.$ $\left[\mathrm{Cu}^{2+}\right]$, donde $\left[\mathrm{Cu}^{2+}\right]$ es la actividad del ión $\mathrm{Cu}^{2+}$ libre), se realizó con un electrodo selectivo de ión $\mathrm{Cu}^{2+}$ (Rachou et al. 2007). La materia orgánica se determinó de acuerdo con Sadzawka et al. (2006). La textura de los suelos se determinó a través del método hidrométrico de Sheldrick y Wang (1993).

Las concentraciones de $\mathrm{Cu}, \mathrm{Zn}, \mathrm{Pb}$ y As se determinaron por espectroscopia de absorción atómica. Los límites de detección fueron los siguientes: $\mathrm{Cu}$,
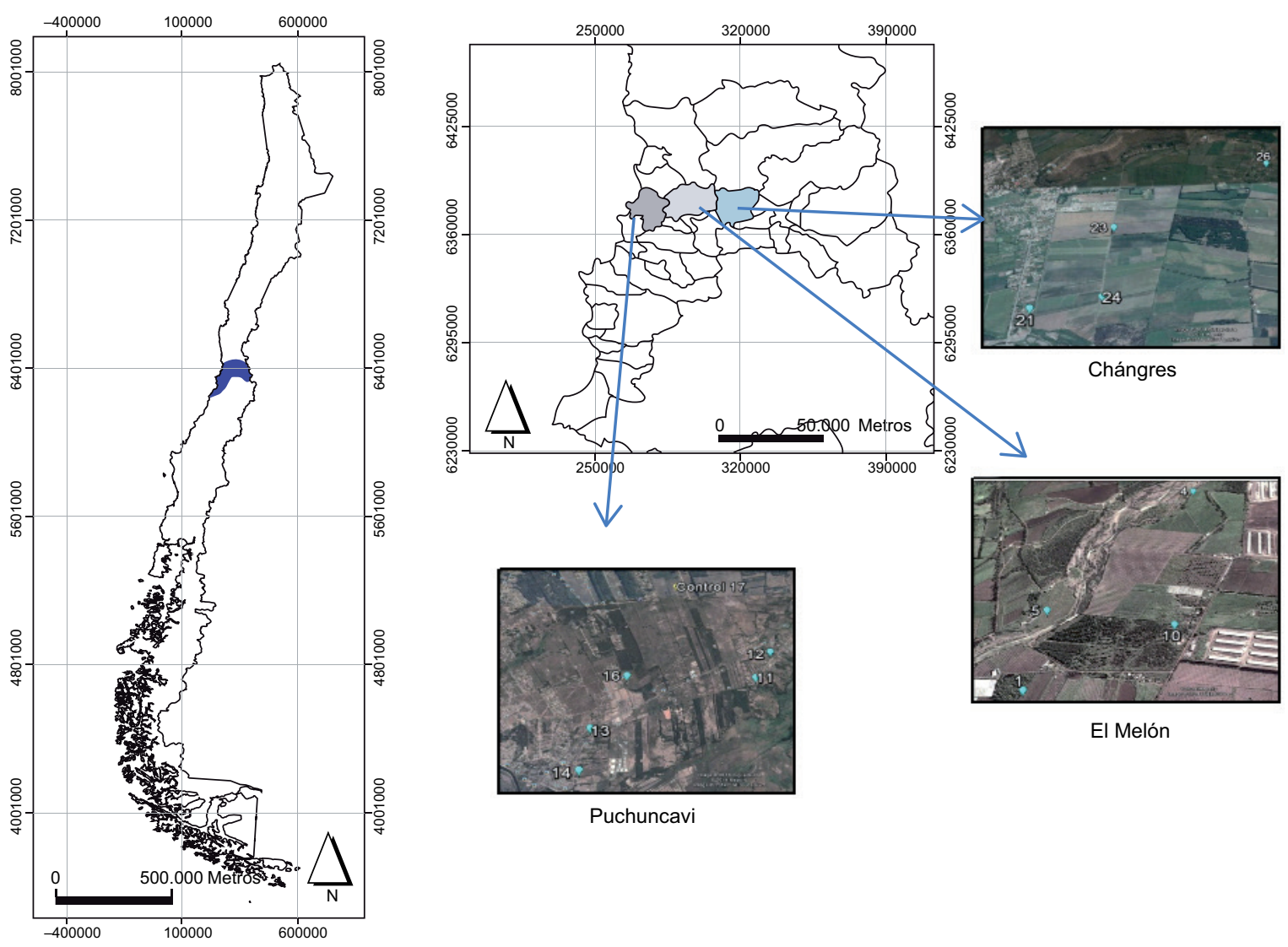

Fig. 1. Localización de sitios de muestreo en la región de Valparaíso, Chile central 
CUADRO I. LOCALIZACIÓN DE LOS SUELOS Y SU USO AGRÍCOLA

\begin{tabular}{|c|c|c|c|c|}
\hline Localidad & Código suelos & Latitud & Longitud & Cultivo \\
\hline El Melón & 1 & $32^{\circ} 39^{\prime} 45.93 " \mathrm{~S}$ & $71^{\circ} 12^{\prime} 35.71^{\prime \prime} \mathrm{W}$ & Zanahoria \\
\hline El Melón & 4 & $32^{\circ} 39^{\prime} 9.65^{\prime \prime} \mathrm{S}$ & $71^{\circ} 12^{\prime} 25.69^{\prime \prime} \mathrm{W}$ & Alfalfa \\
\hline El Melón & 5 & $32^{\circ} 39^{\prime} 35.20^{\prime \prime} \mathrm{S}$ & $71^{\circ} 12^{\prime} 38.29^{\prime \prime} \mathrm{W}$ & Nogales \\
\hline El Melón & 10 & $32^{\circ} 39^{\prime} 33.40^{\prime \prime} \mathrm{S}$ & $71^{\circ} 12^{\prime} 20.50^{\prime \prime} \mathrm{W}$ & Palto \\
\hline Puchuncaví & 11 & $32^{\circ} 44^{\prime} 16.96 " \mathrm{~S}$ & $71^{\circ} 27^{\prime} 11.49^{\prime \prime} \mathrm{W}$ & Barbecho \\
\hline Puchuncaví & 12 & $32^{\circ} 44^{\prime} 4.14^{\prime \prime} \mathrm{S}$ & $71^{\circ} 27^{\prime} 3.79^{\prime \prime} \mathrm{W}$ & Barbecho \\
\hline Puchuncaví & 13 & $32^{\circ} 44^{\prime} 38.21^{\prime \prime} \mathrm{S}$ & $71^{\circ} 28^{\prime} 21.12^{\prime \prime} \mathrm{W}$ & Haba \\
\hline Puchuncaví & 14 & $32^{\circ} 44^{\prime} 55.38^{\prime \prime} \mathrm{S}$ & $71^{\circ} 28^{\prime} 23.65^{\prime \prime} \mathrm{W}$ & Membrillo \\
\hline Puchuncaví & 16 & $32^{\circ} 44^{\prime} 14.28^{\prime \prime} \mathrm{S}$ & $71^{\circ} 28^{\prime} 7.64^{\prime \prime} \mathrm{W}$ & Limón \\
\hline Chagres & 21 & $32^{\circ} 47^{\prime} 41.00^{\prime \prime} \mathrm{S}$ & $70^{\circ} 57^{\prime} 26.40^{\prime \prime} \mathrm{W}$ & Maíz \\
\hline Chagres & 23 & $32^{\circ} 47^{\prime} 37.91^{\prime \prime} \mathrm{S}$ & $70^{\circ} 57^{\prime} 4.11 ” \mathrm{~W}$ & Maíz \\
\hline Chagres & 24 & $32^{\circ} 47^{\prime} 9.97^{\prime \prime} \mathrm{S}$ & $70^{\circ} 57^{\prime} 3.41 " \mathrm{~W}$ & Maíz \\
\hline Chagres & 26 & $32^{\circ} 46^{\prime} 38.32^{\prime \prime} \mathrm{S}$ & $70^{\circ} 56^{\prime} 2.50^{\prime \prime} \mathrm{W}$ & Barbecho \\
\hline
\end{tabular}

$0.017 \mathrm{mg} / \mathrm{kg}$; As, $0.086 \mathrm{mg} / \mathrm{kg}$; Zn, $0.047 \mathrm{mg} / \mathrm{kg}$, y $\mathrm{Pb}, 0.066 \mathrm{mg} / \mathrm{kg}$. Las muestras de suelo se digirieron en ácido nítrico hirviendo, seguido de adición de ácido perclórico (Maxwell 1968). Se utilizaron tubos de refrigeración para evitar la volatilización del As. Para las determinaciones de las concentraciones solubles de $\mathrm{Cu}$ y As, se utilizó una solución de $0.1 \mathrm{M} \mathrm{KNO}_{3}$ como extractante (Stuckey et al. 2008). Los límites de detección fueron: $\mathrm{Cu}, 0.014 \mathrm{mg} / \mathrm{L}, \mathrm{y}$ As, 0.005 $\mathrm{mg} / \mathrm{L}$. La calidad se aseguró mediante la digestión de forma similar en muestras de referencia certificadas: PACS-2 obtenida del Consejo de Investigación Nacional de Canadá, y GRX-2 obtenida de la United States Geological Survey. Los valores obtenidos estuvieron dentro del $10 \%$ del valor certificado. La concentración de metales se expresa como peso seco.

\section{Bioensayo de evasión}

Los bioensayos se llevaron a cabo en recipientes de plástico de un litro de capacidad, los cuales se dividieron en dos secciones con una hoja extraíble de acuerdo con la norma ISO (ISO, 2007). En una sección se colocaron $300 \mathrm{~g}$ de suelo de prueba y en la otra $300 \mathrm{~g}$ de suelo control de una zona sin actividad minera. Los suelos fueron humedecidos antes de la realización de los bioensayos. Por cada suelo de prueba se consideraron cinco réplicas con diez lombrices cada una. Las lombrices se obtuvieron de un centro de lumbricultura Pachamama en la comuna de Quillota (V Región, Chile Central). Las lombrices presentaban el mismo tamaño y madurez sexual. Para iniciar el bioensayo, se extrajo la lámina de plástico y diez lombrices de E. foetida se colocaron en la línea media que separaba las dos secciones. Al final del tiempo de exposición de 48 h, se contó el número de lombrices en cada sección, con un régimen de $16 \mathrm{~h}$ luz $/ 8 \mathrm{~h}$ oscuridad a una temperatura ambiental de $21 \pm 2{ }^{\circ} \mathrm{C}$. Los suelos se consideraron tóxicos cuando el porcentaje de evasión era igual o mayor que $80 \%$. Para evitar que las lombrices se escapasen de los contenedores, se cubrieron con tapas de plástico transparente con agujeros para permitir el intercambio de gases y paso de la luz. La prueba se consideró inválida cuando se observó más de una lombriz por repetición muerta o desaparecida al final del periodo de prueba.

\section{Bioensayo de reproducción}

El bioensayo de reproducción en E. foetida (ISO 2012, OECD 2004) permite determinar el efecto de suelos sobre la reproducción y supervivencia de las lombrices adultas E. foetida. Para el bioensayo se colocaron $500 \mathrm{~g}$ de suelo en recipientes de vidrio experimentales de $750 \mathrm{ml}$. El suelo se humedeció con agua desionizada para alcanzar $60 \%$ de su capacidad de retención de agua. La prueba se realizó a una temperatura controlada de $20^{\circ} \mathrm{C} \pm 2{ }^{\circ} \mathrm{C}$, con iluminación de 400 lx. Al comienzo del bioensayo, las lombrices de tierra se lavaron con agua destilada, se secaron con papel secante y se pesaron. Diez lombrices adultas con clitelo visible (peso de $3.4 \pm 0.1 \mathrm{~g}$ ) se incubaron en cada contenedor y fueron alimentadas con estiércol humedecido. Se consideraron ocho réplicas por cada suelo; el diseño fue aleatorio. El fotoperiodo fue de $12 \mathrm{~h}$ de luz y $12 \mathrm{~h}$ de oscuridad. Después de cuatro semanas de exposición, las lombrices de tierra fueron retiradas del suelo y se determinó la supervivencia, el número de capullos y el número de juveniles.

\section{Análisis estadístico}

Para comparar las variables de respuesta entre los suelos, se aplicó una prueba de Kruskal-Wallis, 
utilizando un nivel de significancia de $5 \%$. Para normalizar los datos, éstos fueron transformados mediante la siguiente fórmula:

$\sqrt{X+0.5}$

Después se realizó un análisis de componentes principales. Todas las pruebas se realizaron usando el programa Minitab 17.

\section{RESULTADOS Y DISCUSIÓN}

Los resultados de las características físicas y químicas de los suelos estudiados, $\mathrm{pH}$, porcentaje de materia orgánica, conductividad eléctrica, granulometría y concentración total de metales ( $\mathrm{As}, \mathrm{Cu}, \mathrm{Zn}$, $\mathrm{Pb}$, solubles de $\mathrm{As} \mathrm{y} \mathrm{Cu}, \mathrm{y} \mathrm{pCu}^{+2}$, pueden observarse respecto a sus valores mínimos, máximos, media y desviación estándar en el cuadro II.

El orden de abundancia de los metales basados en el promedio de concentraciones totales de los metales considerando todos los suelos de las tres localidades fue $\mathrm{Cu}>\mathrm{Zn}>\mathrm{Pb}>$ As.

Las concentraciones de los metales están dentro de los valores reportados por Ávila et al. (2009), de 65 $\mathrm{kg}$ a $1579 \mathrm{mg} / \mathrm{kg}$ de cobre total y 14 a $35 \mathrm{mg} / \mathrm{kg}$ de As total; asimismo, de los encontrados por Aguilar et al. (2011) para el cobre que son de $70 \mathrm{mg} / \mathrm{kg}$ y alrededor de $700 \mathrm{mg} / \mathrm{kg}$ en zonas con y sin actividad minera, respectivamente. A diferencia de estos autores, que

CUADRO II. CONCENTRACIONES DE As, $\mathrm{Cu}, \mathrm{Zn} \mathrm{Pb}$ Y PROPIEDADES FÍSICAS Y QUÍMICAS DE LOS SUELOS DE LAS TRES LOCALIDADES ESTUDIADAS

\begin{tabular}{lcc}
\hline Propiedad del suelo & $\begin{array}{c}\text { Valores } \\
\text { mínimos y } \\
\text { máximos }\end{array}$ & $\begin{array}{c}\text { Media } \\
+/ \text { - desviación } \\
\text { estándar }\end{array}$ \\
\hline Materia orgánica $(\%)$ & $0.7-4.9$ & $3.2 \pm 1.2$ \\
$\mathrm{pH}$ & $6.1-7.6$ & $7.1 \pm 0.4$ \\
Conductividad $(\mathrm{dS} / \mathrm{m})$ & $0.2-1.9$ & $1.2 \pm 0.6$ \\
$\mathrm{pCu}$ & $6.8-9.5$ & $8.5 \pm 0.8$ \\
As soluble $(\mathrm{mg} / \mathrm{L})$ & $0.002-0.097$ & $0.031 \pm 0.030$ \\
$\mathrm{Cu}$ soluble $(\mathrm{mg} / \mathrm{L})$ & $0.039-0.707$ & $0.242 \pm 0.207$ \\
As total $(\mathrm{mg} / \mathrm{kg})$ & $7-34$ & $21.1 \pm 9.7$ \\
$\mathrm{Cu}$ total $(\mathrm{mg} / \mathrm{kg})$ & $100-1295$ & $492.4 \pm 324.8$ \\
$\mathrm{Zn}$ total $(\mathrm{mg} / \mathrm{kg})$ & $94-345$ & $184.5 \pm 66.8$ \\
Pb total $(\mathrm{mg} / \mathrm{kg})$ & $27-97$ & $50.7 \pm 20.7$ \\
Arena $(\%)$ & $25-95$ & $56 \pm 20$ \\
Arcilla $(\%)$ & $5-32$ & $16 \pm 8$ \\
Limo $(\%)$ & $0-43$ & $27 \pm 13$ \\
\hline
\end{tabular}

$\mathrm{pCu}^{+2}$ logaritmo negativo de la actividad de cobre libre $\mathrm{Cu}^{+2}$ también trabajaron con suelos en la misma zona de estudio, el pH en este estudio fue cercano a la neutralidad y la materia orgánica fue menor, lo cual se debe a las diferencias entre puntos y épocas de muestreo. Al comparar las concentraciones de metales totales con la directiva de Kelley en Acevedo et al. (2005) para la clasificación de suelos contaminados, éstos se clasifican de acuerdo con las concentraciones de cobre total en ligera y altamente contaminados, ya que dichas concentraciones van desde los $100 \mathrm{mg} / \mathrm{kg}$. En cuanto a las concentraciones de zinc y plomo en los suelos, éstas corresponden a valores típicos de suelos no contaminados, en tanto que los valores de arsénico representan suelos no contaminados y con contaminación ligera.

La variable de cobre total en el análisis de componentes principales no se asoció con las variables de plomo, arsénico y zinc (Fig. 2), lo cual sugiere que proviene de diferentes fuentes, tales como emisiones de material particulado desde las fundiciones y termoeléctricas cercanas a la zona de estudio, y el uso de plaguicidas con cobre en la actividad agrícola. $\mathrm{Al}$ respecto, se ha estimado que con una tasa recomendada de $3 \mathrm{~kg}$ de cobre aplicados como fungicida (Aguilar et al. 2011), con cuatro aplicaciones por año durante 40 años, en un suelo de $20 \mathrm{~cm}$ de profundidad y con una densidad de $1200 \mathrm{~kg} / \mathrm{m}^{3}$, en ausencia de escorrentía de cobre en el suelo, aproximadamente $50 \%$ de cobre aplicado en los cultivos se acumula en los suelos, en tanto que el resto es retenido por el propio cultivo. En estas condiciones, los fungicidas podrían contribuir a concentraciones de cobre de alrededor de $100 \mathrm{mg} / \mathrm{kg}$.

El porcentaje de sobrevivencia fue de alrededor de $90 \%$ en todos los suelos estudiados. El porcentaje de comportamiento de evasión fue significativamente más alto (superior a $80 \%$ de evasión) en los suelos 5 y 14, donde la toxicidad fue aguda. Por el contrario, los suelos 1 y 26 son los que mostraron menor porcentaje de evasión (inferior a $20 \%$ ). La producción de capullos fue significativamente menor en los suelos 14 y $26(\mathrm{p}<0.05)$. Respecto a la producción de juveniles, a excepción del suelo 14, no hubo diferencias significativas entre las muestras (Cuadro III).

Los análisis químicos de suelos muestran información de las concentraciones y extractabilidad de metales, pero no ofrecen información directa sobre la biodisponibilidad de los contaminantes para la biota (Langdon et al. 2005, Cataldo et al. 2011). Los resultados del presente estudio muestran que la mayoría de los suelos no presentan toxicidad aguda. No se encontraron diferencias significativas en el 


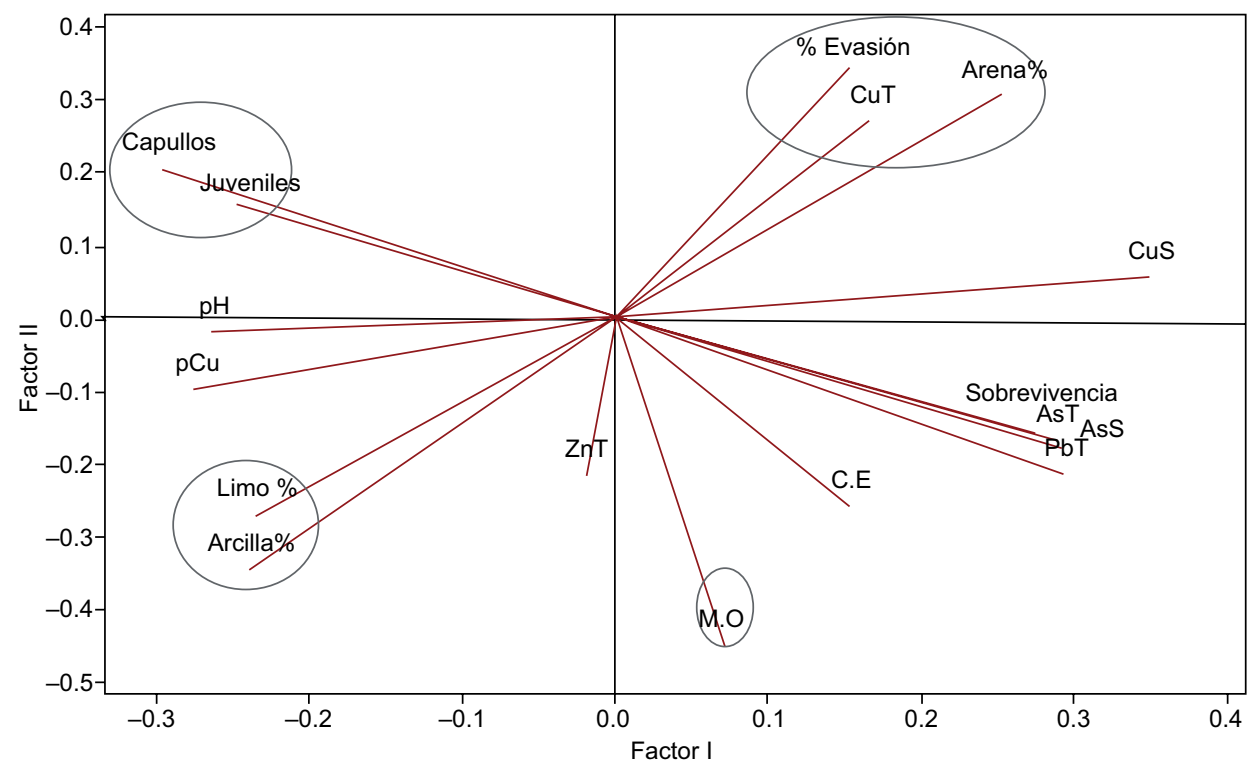

Fig. 2. Distribución de las variables definidas por el análisis de componentes principales: C.E, conductividad eléctrica; M.O., materia orgánica; $\mathrm{pCu}$, actividad de cobre; AsS, arsénico soluble; $\mathrm{CuS}$, cobre soluble; AsT, arsénico total; CuT, cobre total; PbT, plomo total; ZnT, zinc total

CUADRO III. COMPARACIÓN DE LOS RESULTADOS DE LOS BIOENSAYOS CRÓNICOS Y AGUDOS CON E. foetida ENTRE LOS SUELOS

\begin{tabular}{lcccc}
\hline Suelo & $\begin{array}{c}\text { Sobrevivencia } \\
(\%)\end{array}$ & $\begin{array}{c}\text { Evasión } \\
(\%)\end{array}$ & $\begin{array}{c}\text { Cocones } \\
\left(\mathrm{N}^{\circ}\right)\end{array}$ & $\begin{array}{c}\text { Juveniles } \\
\left(\mathrm{N}^{\circ}\right)\end{array}$ \\
\hline 1 & $98 \mathrm{a}$ & $12 \mathrm{ab}$ & $53 \mathrm{abc}$ & $74 \mathrm{ab}$ \\
4 & $92 \mathrm{a}$ & $50 \mathrm{ab}$ & $60 \mathrm{abc}$ & $68 \mathrm{ab}$ \\
5 & $93 \mathrm{a}$ & $92 \mathrm{~b}$ & $61 \mathrm{abc}$ & $53 \mathrm{ab}$ \\
10 & $90 \mathrm{a}$ & $64 \mathrm{ab}$ & $73 \mathrm{ab}$ & $68 \mathrm{ab}$ \\
11 & $100 \mathrm{a}$ & $52 \mathrm{ab}$ & $22 \mathrm{abc}$ & $27 \mathrm{ab}$ \\
12 & $98 \mathrm{a}$ & $56 \mathrm{ab}$ & $39 \mathrm{abc}$ & $76 \mathrm{ab}$ \\
13 & $98 \mathrm{a}$ & $67 \mathrm{ab}$ & $11 \mathrm{bc}$ & $17 \mathrm{ab}$ \\
14 & $97 \mathrm{a}$ & $96 \mathrm{~b}$ & $7 \mathrm{c}$ & $6 \mathrm{~b}$ \\
16 & $96 \mathrm{a}$ & $32 \mathrm{ab}$ & $33 \mathrm{abc}$ & $49 \mathrm{ab}$ \\
21 & $91 \mathrm{a}$ & $44 \mathrm{ab}$ & $72 \mathrm{ab}$ & $84 \mathrm{ab}$ \\
23 & $95 \mathrm{a}$ & $64 \mathrm{ab}$ & $27 \mathrm{abc}$ & $45 \mathrm{ab}$ \\
24 & $95 \mathrm{a}$ & $40 \mathrm{ab}$ & $29 \mathrm{abc}$ & $26 \mathrm{ab}$ \\
26 & $98 \mathrm{a}$ & $16 \mathrm{ab}$ & $10 \mathrm{bc}$ & $19 \mathrm{ab}$ \\
\hline
\end{tabular}

Letras diferentes en la misma columna indican diferencias significativas $(\mathrm{p}<0.05)$

porcentaje de evasión entre suelos, con excepción de dos de ellos que presentaron las concentraciones más altas de cobre total. Respecto a la sobrevivencia y reproducción, sus valores concuerdan con los resultados reportados por Ávila et al. (2009), quienes no observaron efectos en la sobrevivencia de $E$. foetida a concentraciones similares encontradas en el presente estudio, lo cual sugiere que los agentes químicos en estos suelos no están en concentraciones biodisponibles o no son suficientes para provocar un efecto sobre la selección de hábitat, sobrevivencia y reproducción de $E$. foetida.

Respecto a las concentraciones de cobre en los suelos, éstas fueron menores a las descritas como tóxicas para lombrices de acuerdo con Barros-Amorim y Scott-Fordsmand (2012), quienes reportaron que la toxicidad de $\mathrm{CuCl}_{2}$ en ensayos de comportamiento de evasión tuvo un EC50 de 400 (378-573) $\mathrm{mgCu} / \mathrm{kg}$, valor superior al encontrado en la mayoría de las muestras del presente estudio. Otra variable que puede explicar la baja toxicidad es la capacidad de las lombrices para inducir la producción de proteínas que ligan cationes libres de metales, como es el caso del cobre, denominadas metalotioneinas (Mosleh et al. 2007). Al respecto, Cataldo et al. (2011), reportaron una correlación entre las concentraciones de cobre y metalotioneinas en E. foetida expuesta a suelos con similares niveles de concentraciones de cobre que en el presente estudio.

La menor producción de capullos se observó en el suelo que presentó una concentración de $898 \mathrm{mg} /$ $\mathrm{kg}$ de cobre total (Cuadro III), lo cual difiere de lo reportando por Zhou et al. (2013), quienes encontraron un efecto sobre la producción de capullos en una menor concentración de cobre total $(50 \mathrm{mg} / \mathrm{kg})$. Estas diferencias se deben probablemente a que estos autores utilizaron suelos con $\mathrm{pH}$ más ácido y menor contenido de materia orgánica. Al respecto, se sabe 
que a pH ácido y bajo contenido de materia orgánica aumenta la biodisponibilidad de metales en suelos (Ávila et al. 2009). Van Gestel et al. (2009) encontraron efectos de suelos contaminados con metales en lombrices a nivel celular, pero no a nivel de organización superior, como abundancia y diversidad de especies. Estos autores atribuyen la respuesta anterior a las características de los suelos que presentaron $\mathrm{pH}$ alcalino y altos niveles de materia orgánica. De ahí que no necesariamente altas concentraciones de metales generen efectos tóxicos, al igual que se reporta en el presente estudio.

Por otra parte, la baja toxicidad de los suelos estudiados concuerda con los resultados obtenidos por Spurgeon et al. (1994), quienes encontraron una CL50-56-días para E. fetida de $555 \mathrm{mgCu} / \mathrm{kg}$ y $745 \mathrm{mgZn} / \mathrm{kg}$ en suelo artificial acorde con la clasificación de la Organización de Cooperación y Desarrollo Económicos (OECD, por sus siglas en inglés). Neuhauser et al. (1985) reportaron una CL50-14-días de $643 \mathrm{mg} \mathrm{Cu} / \mathrm{kg}$ y $662 \mathrm{mgZn} / \mathrm{kg}$ en suelo artificial. Aunado a lo anterior, la baja toxicidad de los suelos en el presente estudio podría deberse a la capacidad de las lombrices de regular internamente los metales como zinc y cobre (Tiago Natal-da-Luz et al. 2014).

En el análisis de componentes principales, el primer factor explica $41 \%$ y el segundo factor $24 \%$ de la varianza total. En el primer factor, las variables con mayor carga fueron textura, cobre soluble, arsénico y porcentaje de evasión (Cuadro IV). La distribución de las variables en los dos factores se observa en la figura 2, donde se identifican tres grupos: $a$ ) características granulométricas, $b$ ) evasión, arena (\%) y cobre total, c) plomo total, arsénico total y soluble. También se destaca aisladamente la materia orgánica (M.O.). No obstante la ausencia de diferencias significativas en la toxicidad entre suelos ( $p>0.05)$, la concentración de cobre total es la variable que más se relaciona con la variación en el porcentaje de evasión de E. foetida entre suelos, lo que concuerda con la relación encontrada por Ávila et al. (2007) en la variable de reproducción. Lo anterior sugiere que el cobre podría ser un factor que afecta la selección del hábitat de las lombrices de tierra.

Por otra parte, la variable materia orgánica contribuyó a disminuir el efecto tóxico potencial de los metales, ya que en el presente estudio se relacionó inversamente con el comportamiento de evasión, sugiriendo un efecto protector. Otro factor que pudo haber reducido la biodisponibilidad fue el contenido de arcilla, debido a su alta capacidad de intercambio catiónico.
CUADRO IV. CARGAS DE LAS VARIABLES FÍSICAS, QUÍMICAS Y BIOLÓGICAS EN LOS DOS PRIMEROS FACTORES DEFINIDAS EN EL ANÁLISIS DE COMPONENTES PRINCIPALES

\begin{tabular}{lrr}
\hline Variable & Factor I & Factor II \\
\hline Evasión (\%) & 0.15 & 0.34 \\
Sobrevivencia (\%) & 0.28 & -0.16 \\
Cocones (N $)$ & -0.30 & 0.20 \\
C.E $(\mathrm{dS} / \mathrm{m})$ & 0.15 & -0.26 \\
M.O (\%) & 0.07 & -0.45 \\
pH & -0.26 & -0.02 \\
pCu & -0.28 & -0.10 \\
AsS (mg/L) & 0.29 & -0.18 \\
CuS (mg/L) & 0.35 & 0.06 \\
AsT (mg/kg) & 0.29 & -0.17 \\
CuT (mg/kg) & 0.17 & 0.27 \\
PbT (mg/kg) & 0.29 & -0.21 \\
ZnT (mg/kg) & -0.02 & -0.22 \\
Arena $(\%)$ & 0.25 & 0.31 \\
Arcilla (\%) & -0.24 & -0.35 \\
Limo $(\%)$ & -0.23 & -0.27 \\
Juveniles $\left(N^{\circ}\right)$ & -0.25 & 0.16 \\
\hline
\end{tabular}

C.E.: conductividad eléctrica; M.O.: materia orgánica; $\mathrm{pCu}$ : actividad de cobre; AsS: arsénico soluble; CuS: cobre soluble; AsT: arsénico total; CuT: cobre total; PbT: plomo total; ZnT: zinc total

\section{CONCLUSIÓN}

La mayoría de los suelos estudiados no contiene agentes químicos en concentraciones biodisponibles que provoquen toxicidad, tanto aguda como crónica, en E. foetida. Sin embargo, la concentración de cobre total fue la variable que más se relacionó con el porcentaje de evasión. Las variables de comportamiento de evasión y reproducción se relacionaron inversamente. Es decir, los suelos con mayor evasión tuvieron menor reproducción. Se sugiere el uso de bioensayos de comportamiento de evasión como alternativa a los de reproducción, ya que permiten tener respuestas de manera más temprana.

\section{AGRADECIMIENTOS}

Este estudio fue financiado por el Fondo Nacional de Desarrollo Científico y Tecnológico. Proyecto FONDECYT Núm. 1130041.

\section{REFERENCIAS}

Acevedo E., Carrasco M., León O., Silva P., Castillo G., Ahumada I., Borie G. y González S. (2005). Informe de 
criterios de calidad de suelo agrícola. Servicio Agrícola y Ganadero, Chile, 205 pp.

Aguilar R., Hormazábal C., Gaete H. y Neaman A. (2011). Spatial distribution of copper, organic matter and $\mathrm{pH}$ in agricultural soils affected by mining activities. J. Soil Sci. Plant Nutr. 11 (3), 125-144. DOI: $10.4067 / \mathrm{S} 0718-95162011000300010$

Ávila G., Gaete H., Morales M. y Neaman A. (2007). Reproducción de Eisenia fetida en suelos agrícolas de áreas mineras contaminadas por cobre y arsénico. Pesqui. Agropecu. Bras. 42 (3), 435-441. DOI: $10.1590 / \mathrm{S} 0100-204 \mathrm{X} 2007000300018$

Ávila G., Gaete H., Sauvé S. y Neaman A. (2009). Materia orgánica reduce la roxicidad del robre para la rombriz Eisenia foetida en ruelos de áreas mineras en Chile central. Chilean J. Agric. Res. 69 (2), 252-259.

DOI: $10.4067 / \mathrm{S} 0718-58392009000200015$

Barros-Amorim J. y Scott-Fordsmand J. (2012). Toxicity of copper nanoparticles and $\mathrm{CuCl}_{2}$ salt to Enchytraeus albidus worms: Survival, reproduction and avoidance responses. Environ. Pollut. 164, 164-168. DOI: 10.1016/j.envpol.2012.01.015

Cataldo J., Hidalgo M., Neaman A. y Gaete H. (2011). Use of molecular biomarkers in Eisenia foetida to assess copper toxicity in agricultural soils affected by mining activities. J. Soil Sci. Plant Nutr. 11 (3), 57-70. DOI 10.4067/S0718-95162011000300005

Curry J. y Schmidt O. (2007). The feeding ecology of earthworms-A review Pedobiologia 50 (6), 463-477. DOI: 10.1016/j.pedobi.2006.09.001

Ferreira-Cravo M., Ventura-Lima J., Zomer J., Amado L., Geracitano L., Rebelo M., Bianchinia A. y Monserrat J. (2009). Antioxidant responses in different body regions of the polychaeta Laeonereis acuta (Nereididae) exposed to copper. Ecotox. Environ. Safe. 72 (2), 388393. DOI:10.1016/j.ecoenv.2008.07.003

Ginocchio R., Cárcamo V., Bustamante E., Trangolao E., De la Fuente L. y Neaman A. (2013). Efficacy of fresh and air-dried biosolids as amendments for remediation of acidic and metal-polluted soils: A short-term laboratory assay. J. Soil Sci. Plant Nutr. 13 (4), 855-869. DOI:10.4067/S0718-95162013005000068

González I., Neaman A., Rubio P. y Cortés A. (2014). Spatial distribution of copper and $\mathrm{pH}$ in soils affected by intensive industrial activities in Puchuncaví and Quintero, central Chile. J. Soil Sci. Plant Nutr. 14 (4), 943-953. DOI: $10.4067 /$ S0718-95162014005000074

Hormazábal C., Aguilar R., Cisternas M. y Neaman A. (2013). Modelo predictivo de la distribución espacial de cobre en suelos agrícolas de la cuenca del Río Aconcagua, Chile. Investig. Geogr. Chile (46), 79-92.

ISO (2007). ISO-17512-1-2007. Soil quality - Avoidance test for determining the quality of soils and effects of chemicals on behaviour - Part 1: Test with earthworms (Eisenia fetida and Eisenia andrei). International Organization for Standardization, Ginebra, Suiza, 26 pp.

ISO (2012). ISO-11268-2-2012. Soil quality effects of pollutants on earthworms. Part 2: Determination of effects on reproduction of Eisenia fetida/Eisenia andrei. International Organization for Standardization, Ginebra, Suiza, 21 pp.

Langdon C., Hodson M., Arnold R. y Black S. (2005). Survival, $\mathrm{Pb}$-uptake and behaviour of three species of earthworm in $\mathrm{Pb}$ treated soils determined using an OECD-style toxicity test and a soil avoidance test. Environ. Pollut. 138 (2), 368-375.

DOI: 10.1016/j.envpol.2005.03.002

Maxwell J. A. (1968). Rock and mineral analysis. Interscience Publishers, Nueva York, 584 pp.

Mosleh Y., Paris-Palacios S., Ahmed M., Mahmoud F., Osman F. y Biagianti-Risbourg S. (2007). Effects of chitosan on oxidative stress and metallothioneins in aquatic worm Tubifex tubifex (Oligochaeta, Tubificidae). Chemosphere 67 (1), 167-175.

DOI: 10.1016/j.chemosphere.2006.09.019

MOP (2004). Diagnóstico y clasificación de los cursos y cuerpos de agua según objetivos de calidad: Cuenca del Río Aconcagua. Ministerio de Obras Públicas de Chile, 154 pp. [en línea]. http://www.sinia.cl/1292/ articles-31018 Aconcagua.pdf 09/08/2015

Navarro-Aviñó J., Alonso I. y López-Moya J. (2007). Aspectos bioquímicos y genéticos de la tolerancia y acumulación de metales pesados en plantas. Ecosistemas 16 (2), 10-25.

Neuhauser E., Loehr R., Milligan D. y Malecki M. (1985). Toxicity of metals to the earthworm Eisenia fetida. Biol. Fert. Soils. 1 (3), 149-152.

OECD (2004). Guidelines for the testing of chemicals No 222. Earthworm reproduction test (Eisenia fetida/Eisenia andrei). Organisation for Economic Co-operation and Development. París, Francia, 18 pp.

DOI: $10.1787 / 207$

Rachou J., Gagnon C. y Sauvé S. (2007). Use of an ionselective electrode for free copper measurements in low salinity and low ionic strength matrices. Environ. Chem. 4 (2), 90-97. DOI: 10.1071/EN06036

Rodríguez M., De Arana C., Ramos-Miras J., Gil C. y Boluda R. (2015). Impact of 70 years urban growth associated with heavy metal pollution. Environ. Pollut. 196, 156-163. DOI: 10.1016/j.envpol.2014.10.014

Sadzawka A., Carrasco M., Grez R., Mora M., Flores H. y Neaman A. (2006). Methods of soil analysis recommended for soils of Chile. Commission standards and accreditation. Chilean Society of Soil Science, Santiago, Chile, 164 pp. 
Sheldrick B. y Wang. C. (1993). Particle size distribution. En: Soil sampling and methods of analysis (M. Carter, Ed.). Canadian society of soil science, Lewis Publishers, Boca Raton, FL pp. 499-511.

Spurgeon D., Hopkin S. y Jones D. (1994). Effects of cadmium, copper, lead and zinc on growth, reproduction and survival of the earthworm Eisenia fetida (Savigny): assessing the environmental impact of point-source metal contamination in terrestrial ecosystems. Environ. Pollut. 84 (2), 123-130.

DOI: $10.1016 / 0269-7491(94) 90094-9$

Stuckey J.W., Neaman A., Ravella R., Komarneni S. y Martínez C.E. (2008). Highly charged swelling mica reduces free and extractable $\mathrm{Cu}$ levels in $\mathrm{Cu}-$ contaminated soils. Environ. Sci. and Technol. 42 (24), 9197-9202. DOI: 10.1021/es801799s

Tiago Natal-da-Luz R., Sousa J., Colonese J., Bidone E., Castilhos Z., Egler S. y Polivanov H. (2014). Disposal of dredged sediments in tropical soils: ecotoxicological effects on earthworms. Environ. Monit. Assess. 186 (3), 1487-1497.

DOI: $10.1007 / \mathrm{s} 11356-014-3559-3$
Udovic M. y Lestan D. (2010). Eisenia fetida avoidance behavior as a tool for assessing the efficiency of remediation of $\mathrm{Pb}, \mathrm{Zn}$ and $\mathrm{Cd}$ polluted soil. Environ. Pollut. 158 (8), 2766-2772.

DOI: 10.1016/j.envpol.2010.04.015

Van Gestel C., Koolhaas J., Hamers T., Van Hoppe M., Van Roovert M., Korsman C. y Reinecke S. (2009). Effects of metal pollution on earthworm communities in a contaminated floodplain area: Linking biomarker, community and functional responses. Environ. Pollut. 157 (3), 895-903.

DOI: 10.1016/j.envpol.2008.11.002

Zhou C., Wang Y., Li C. C., Sun R., Yu Y. y Zhou D. (2013). Subacute toxicity of copper and glyphosate and their interaction to earthworm (Eisenia fetida). Environ. Pollut. 180, 71-77.

DOI: $10.1016 /$ j.envpol.2013.05.016

Žižek S. y Zidar P. (2013). Toxicity of the ionophore antibiotic lasalocid to soil-dwelling invertebrates: Avoidance tests in comparison to classic sublethal tests. Chemosphere 92 (5), 570-575.

DOI: 10.1016/j.chemosphere.2013.04.007 